\title{
LRS Bianchi Type II Inflationary Universe with Massless Scalar Field
}

\author{
Raj Bali and Laxmi Poonia \\ Department of Mathematics, University of Rajasthan, Jaipur 302004, India \\ Correspondence should be addressed to Raj Bali, balir5@yahoo.co.in \\ Received 5 September 2011; Accepted 16 October 2011 \\ Academic Editor: P. A. Hughes
}

Copyright ( $) 2011$ R. Bali and L. Poonia. This is an open access article distributed under the Creative Commons Attribution License, which permits unrestricted use, distribution, and reproduction in any medium, provided the original work is properly cited.

Inflationary scenario in locally rotationally symmetric (LRS) Bianchi Type II space-time with massless scalar field with flat potential is discussed. To get the deterministic solution in terms of cosmic time $t$, we have assumed that the scale factor $\sim e^{3 H t}$, that is, $R^{2} S \sim e^{3 H t}$ and $V(\phi)=$ constant where $V$ is effective potential and $\phi$ is Higg's field. We find that spatial volume increases with time and the model isotropizes for large value of $t$ under special condition. The Higg's field decreases slowly and tends to a constant value when $t \rightarrow \infty$. The model represents uniform expansion but accelerating universe and leads to de-Sitter type metric.

\section{Introduction}

Inflationary universes provide a potential solution to the formation of structure problem in Big-Bang cosmology like Horizon problem, Flatness problem, and magnetic monopole problem. Guth [1] introduced the concept of inflation while investigating the problem of why we see no magnetic monopole today. He found that a positive-energy false vacuum generates an exponential expansion of space according to general relativity. In Guth inflationary universe, the scalar field is assumed to start at $\phi=0=\dot{\phi} ; \phi=0$ being a local minimum of $V(\phi)$ where $V$ is effective potential and $\phi$ is Higg's field which breaks the symmetry. In this case, the energy-momentum tensor of particles $\sim T^{4}$ ( $T$ being absolute temperature) almost vanishes in the course of expansion of the universe and the total energy-momentum tensor reduces to vacuum energy, that is, $T^{i j}=g_{i j} V(0)$ where $V(0)$ is effective potential at vanishing temperature (Zel'dovich \& Khlokov [1978]). This leads to

$$
a^{3}=e^{3 H t},
$$

where $a$ is scale factor and $H$ is Hubble constant. Rothman and Ellis [2] have pointed out that we can have a solution of isotropic problem if we work with anisotropic metric and these metrics can be isotropized under various general circumstances. Stein-Schabes [3] has shown that inflation will take place if effective potential $V(\phi)$ has flat region while Higg's field $(\phi)$ evolves slowly but the universe expands in an exponential way due to vacuum field energy. Burd [4] has discussed inflationary scenario in FRW (Friedmann-Robertson-Walker) model. Anninos et al. [5] discussed the significance of inflation for isotropization of universe. In modern cosmology, inflation is an essential ingredient. During the inflationary epoch, the scale factor of the universe grew exponentially allowing a small causally coherent region to grow enough to be identified with the present observable universe. Linde [6] proposed a chaotic model with an assumption that the present universe is originated from chaotic distribution of initial scalar field when potential energy of the field dominates over that of kinetic energy. Later on, it has been shown by Bunn et al. [7] that chaotic scenario can be realized even when scalar field is kinetic energy dominated. Paul et al. [8] have shown that Linde's chaotic scenario is fairly general and can be accommodated even if universe is anisotropic. Bali and Jain [9] has discussed inflationary scenario in LRS Bianchi Type I space-time in the presence of massless scalar field with flat potential. Reddy et al. [10] have investigated inflationary scenario in Kantowski-Sachs spacetime. Recently Bali [11] investigated inflationary scenario in anisotropic Bianchi Type I space-time with flat potential 
considering the scale factor $=e^{3 H t}$ as introduced by Kirzhnits and Linde [12].

Motivated by the above-mentioned research works, we have investigated inflationary scenario in LRS Bianchi Type II space-time with flat potential, and assuming the condition scale factor $a \sim e^{H t}, H$ is Hubble constant as introduced by Kirzhnits and Linde [12]. The model represents an anisotropic universe which isotropizes for large value of $t$ under special condition as shown by Rothman and Ellis [2]. The model represents uniform expansion but accelerating universe. The model leads to de-Sitter space-time.

\section{Metric and Field Equations}

We consider Bianchi Type II metric in the form

$$
d s^{2}=\eta_{a b} \theta^{a} \theta^{b}
$$

with

$$
\theta^{2}=S(d y-x d z)
$$

Therefore, the metric (2) leads to the form

$$
d s^{2}=-d t^{2}+R^{2} d x^{2}+S^{2}(d y-x d z)^{2}+R^{2} d z^{2},
$$

where $R$ and $S$ are functions of $t$-alone.

The Lagrangian is that of gravity minimally coupled to be a scalar field $V(\phi)$ given by

$$
L=\int \sqrt{-g}\left(R-\frac{1}{2} g^{i j} \partial_{i} \phi \partial_{j} \phi-V(\phi)\right) d^{4} x
$$

(Notations have their usual meaning and in geometrized unit $G=c=1)$. Now from the variation of $L$ with respect to the dynamical fields, we obtain Einstein field equation for massless scalar field $V(\phi)$ as

$$
R_{i j}-\frac{1}{2} R g_{i j}=-8 \pi T_{i j},
$$

where

$$
T_{i j}=\partial_{i} \phi \partial_{j} \phi-\left[\frac{1}{2} \partial_{\rho} \phi \partial^{\rho} \phi+V(\phi)\right] g_{i j}
$$

with

$$
\frac{1}{\sqrt{-g}} \partial_{i}\left[\sqrt{-g} \partial_{i} \phi\right]=-\frac{d V}{d \phi},
$$

where $v^{i}$ the flow vector, $\phi$ the Higg's field, $V$ the potential, and $g_{i j}$ the metric tensor. Here

$$
\partial_{i} \phi=\frac{\partial \phi}{\partial x^{i}}, \quad \partial^{\rho} \phi=g^{\rho \ell} \frac{\partial \phi}{\partial x^{\ell}} .
$$

The Einstein's field equation (6) for the metric (4) leads to

$$
\begin{gathered}
\frac{R_{44}}{R}+\frac{S_{44}}{S}+\frac{R_{4} S_{4}}{R S}+\frac{S^{2}}{4 R^{4}}=-8 \pi\left[\frac{1}{2} \dot{\phi}^{2}-V(\phi)\right], \\
\frac{2 R_{44}}{R}+\frac{R_{4}^{2}}{R^{2}}-\frac{3 S^{2}}{4 R^{4}}=-8 \pi\left[\frac{1}{2} \dot{\phi}^{2}-V(\phi)\right], \\
\frac{R_{4}^{2}}{R^{2}}+\frac{2 R_{4} S_{4}}{R S}-\frac{1}{4} \frac{S^{2}}{R^{4}}=8 \pi\left[\frac{1}{2} \dot{\phi}^{2}+V(\phi)\right] .
\end{gathered}
$$

Equations (8) for scalar field leads to

$$
\phi_{44}+\left(\frac{2 R_{4}}{R}+\frac{S_{4}}{S}\right), \quad \phi_{4}=-\frac{d V}{d \phi} .
$$

\section{Solution of Field Equations}

To get deterministic solution in terms of cosmic time $t$, we assume that scale factor $\sim e^{3 H t}$, that is,

$$
R^{2} S \sim e^{3 H t}, \quad H \text { is Hubble constant }
$$

as considered by Bali [11]. We also assume that effective potential $V(\phi)=$ constant. Thus (11) leads to

$$
\phi_{44}+\left(\frac{2 R_{4}}{R}+\frac{S_{4}}{S}\right) \phi_{4}=0 .
$$

Equation (10) leads to

$$
\frac{R_{44}}{R}+\frac{S_{44}}{S}+\frac{3 R_{4} S_{4}}{R S}+\frac{R_{4}^{2}}{R^{2}}=k,
$$

where

$$
16 \pi V(\phi)=k(\text { constant }) .
$$

From (12), we have

$$
\frac{2 R_{4}}{R}+\frac{S_{4}}{S}=3 H
$$

Using (16) in (14), we get

$$
\frac{R_{44}}{R}-\frac{R_{4}^{2}}{R^{2}}+\frac{3 H R_{4}}{R}=\beta,
$$

where

$$
\beta=9 H^{2}-k
$$

From (17), we have

$$
\frac{R_{4}}{R}=\frac{\beta}{3 H}+\gamma e^{-3 H t},
$$

$\gamma$ being constant of integration. Equation (19) leads to

$$
R=\ell e^{\beta t / 3 H} \exp \left(-\frac{\gamma}{3 H} e^{-3 H t}\right) .
$$

Equations (19) and (16) lead to

$$
S=\frac{1}{\ell^{2}} e^{(3 H-(2 \beta / 3 H)) t} \exp \left(\frac{2 \gamma}{3 H} e^{-3 H t}\right) .
$$

Hence the metric (4) reduces to the form

$$
\begin{aligned}
d s^{2}= & -d t^{2}+\ell^{2} e^{2 \beta t / 3 H} \exp \left\{2\left(-\frac{\gamma}{3 H} e^{-3 H t}\right)\right\} d x^{2} \\
& +\frac{1}{\ell^{4}} e^{(6 H-(4 \beta / 3 H)) t} \exp \left\{2\left(-\frac{2 \gamma}{3 H} e^{-3 H t}\right)\right\}(d y-x d z)^{2} \\
& +\ell^{2} e^{2 \beta t / 3 H} \exp \left\{2\left(-\frac{\gamma}{3 H} e^{-3 H t}\right)\right\} d z^{2} .
\end{aligned}
$$


To determine Higg's field $\phi$. Using the assumed condition $V(\phi)=$ constant in (11), we have

$$
\phi_{44}+\left(\frac{2 R_{4}}{R}+\frac{S_{4}}{S}\right) \phi_{4}=0 .
$$

Equation (23) leads to

$$
\phi=-\frac{a e^{-3 H t}}{3 H}+b,
$$

where $a$ and $b$ are constants.

\section{Some Physical and Geometrical Aspects}

The spatial volume $\left(V^{3}\right)$ for the model (22) is given by

$$
V^{3}=R^{2} S=e^{3 H t} .
$$

The expansion $(\theta)$ is given by

$$
\theta=\left(\frac{2 R_{4}}{R}+\frac{S_{4}}{S}\right)=3 H .
$$

The shear $(\sigma)$ is given by

$$
\sigma=\frac{1}{\sqrt{3}}\left(\frac{R_{4}}{R}-\frac{S_{4}}{S}\right),
$$

which leads to

$$
\sigma=\frac{1}{\sqrt{3}}\left(-3 H+3 \gamma e^{-3 H t}\right)+\frac{\beta}{H} .
$$

The deceleration parameter $(q)$ is given by

$$
q=-\frac{\ddot{V} / V}{\dot{V}^{2} / V^{2}}=-1,
$$

where

$$
\frac{\dot{V}}{V}=\frac{V_{4}}{V} ; \quad \frac{\ddot{V}}{V}=\frac{V_{44}}{V} .
$$

\section{Conclusions}

The spatial volume increases with time. Hence inflationary scenario exists in Bianchi Type II space-time. The model (22) in general represents an anisotropic universe. However the model isotropizes for large values of $t$ and $\beta=3 H^{2}$. The Higg's field decreases slowly, and it tends to a constant value when $t \rightarrow \infty$. There is uniform expansion and deceleration parameter $q=-1$. Hence the model leads to de-Sitter spacetime, and the model represents accelerating universe.

\section{References}

[1] A. H. Guth, "Inflationary universe: a possible solution to the horizon and flatness problems," Physical Review D, vol. 23, no. 2, pp. 347-356, 1981.

[2] T. Rothman and G. F. R. Ellis, "Can inflation occur in anisotropic cosmologies?" Physics Letters B, vol. 180, no. 1-2, pp. 19-24, 1986.
[3] J. A. Stein-Schabes, "Inflation in spherically symmetric inhomogeneous models," Physical Review D, vol. 35, no. 8, pp. 2345-2351, 1987.

[4] A. Burd, "Inflation in open FLRW universes," Classical and Quantum Gravity, vol. 10, no. 8, pp. 1495-1505, 1993.

[5] P. Anninos, R. A. Matzner, T. Rothman, and M. P. Ryan Jr., "How does inflation isotropize the universe?" Physical Review $D$, vol. 43, no. 12, pp. 3821-3832, 1991.

[6] A. D. Linde, "Chaotic inflation with constrained fields," Physics Letters B, vol. 202, no. 2, pp. 194-197, 1988.

[7] E. F. Bunn, A. R. Liddle, and M. White, "Four-year COBE normalization of inflationary cosmologies," Physical Review D, vol. 54, no. 10, pp. R5917-R5921, 1996.

[8] B. C. Paul, D. P. Datta, and S. Mukherjee, "Chaotic inflationary scenario in an anisotropic universe," Modern Physics Letters, vol. 1, p. 149, 1986.

[9] R. Bali and V. C. Jain, "Bianchi type I inflationary universe in general relativity," Pramana, vol. 59, no. 1, pp. 1-7, 2002.

[10] D. R. K. Reddy, K. S. Adhav, S. D. Katore, and K. S. Wankhade, "Kantowaski-Sachs inflationary universe in general relativity," International Journal of Theoretical Physics, vol. 48, no. 10, pp. 2884-2888, 2009.

[11] R. Bali, "Inflationary scenario in bianchi type I space-time," International Journal of Theoretical Physics, vol. 50, no. 10, pp. 3043-3048, 2011.

[12] D. A. Kirzhnits and A. D. Linde, "Symmetry behavior in gauge theories," Annals of Physics, vol. 101, no. 1, pp. 195-238, 1976. 

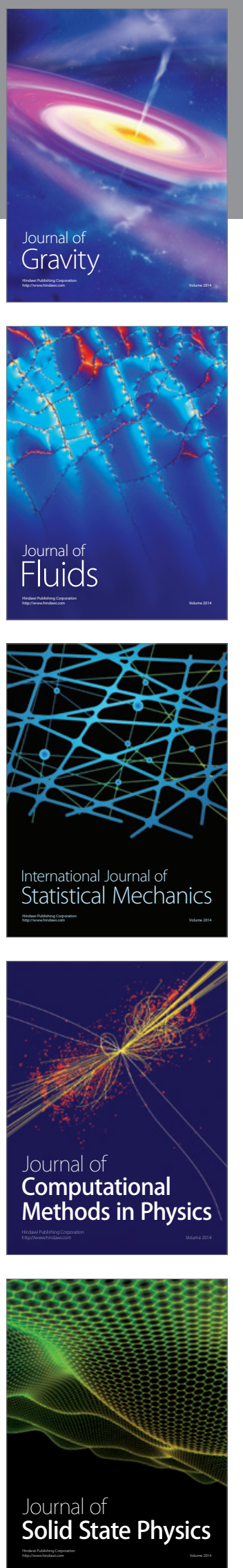

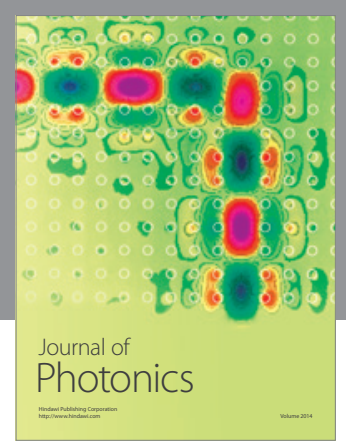

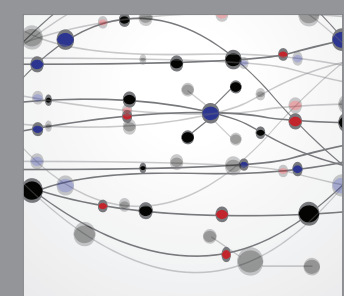

The Scientific World Journal
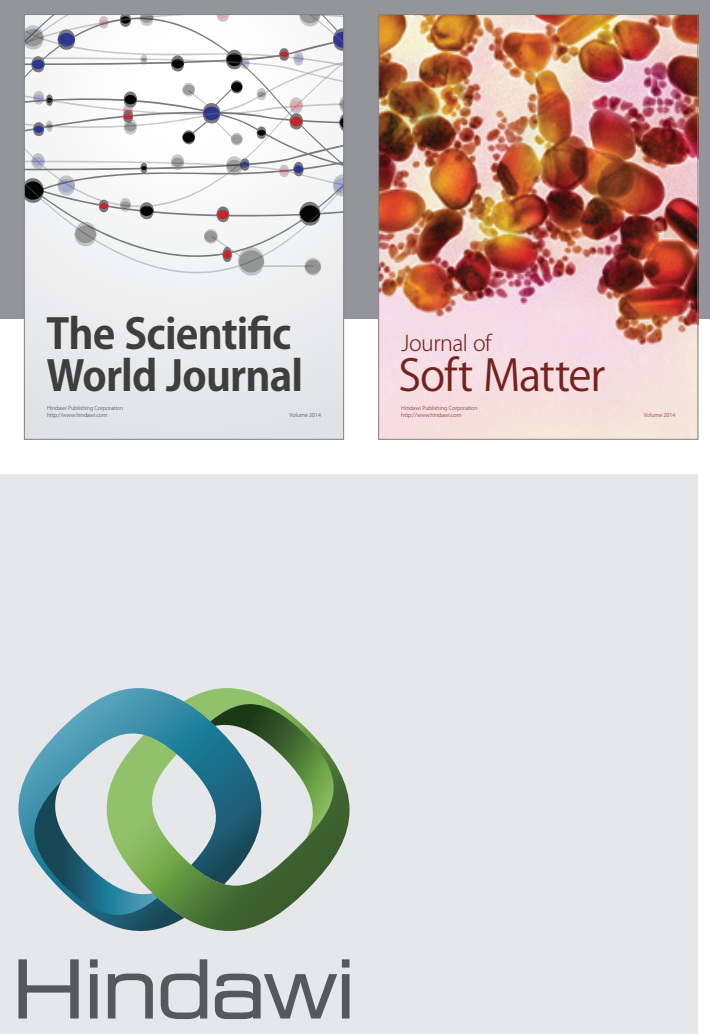

Submit your manuscripts at

http://www.hindawi.com
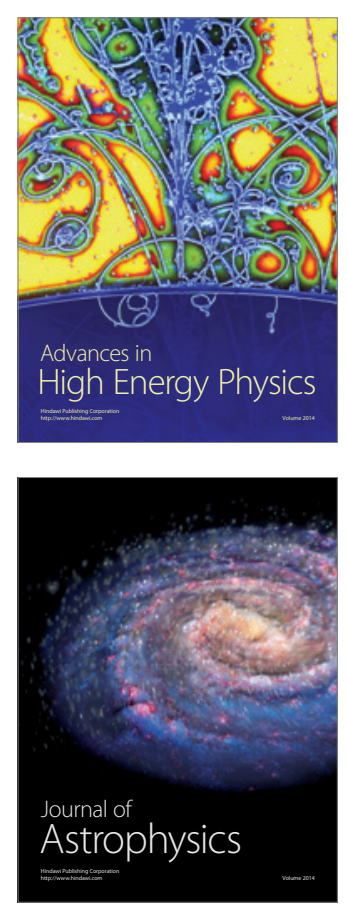
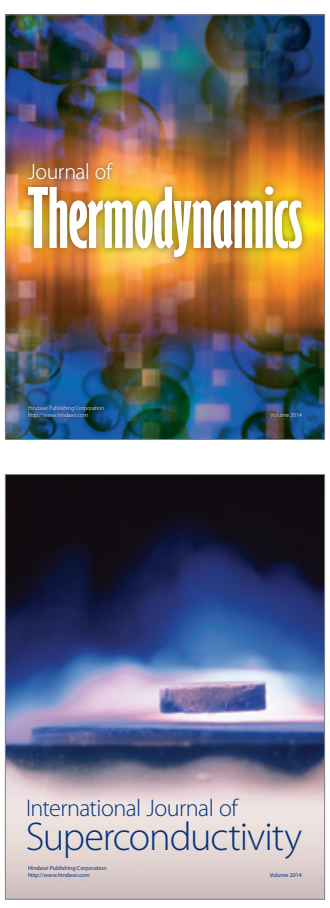
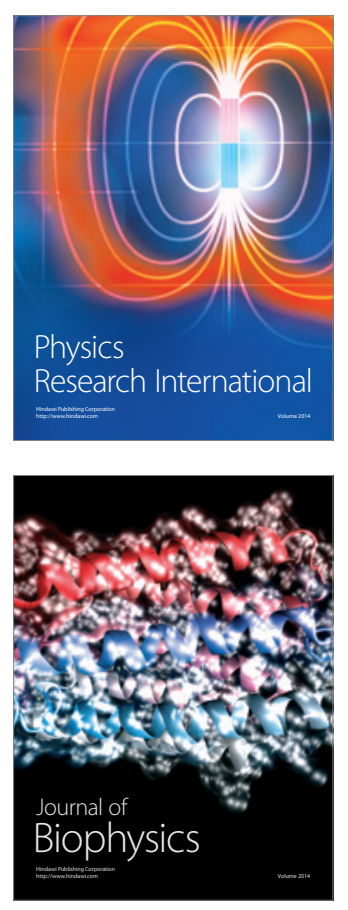
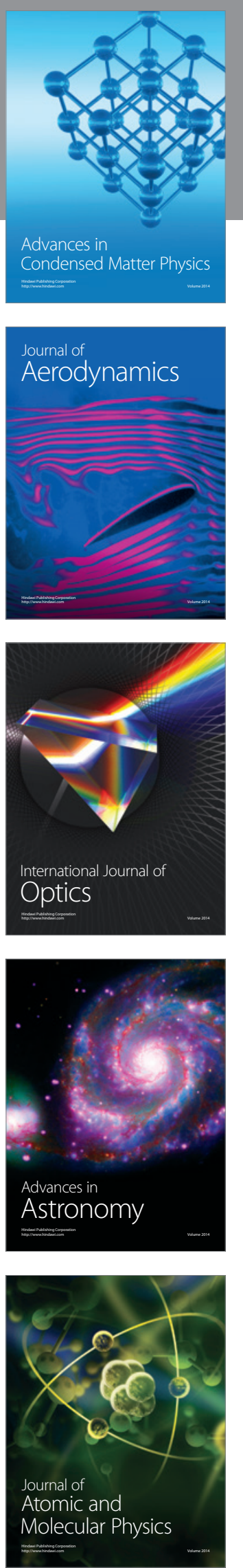